\title{
Student interactions during class activities: a mathematical model.
}

\author{
D. Brunetto*, C. Andrà, N. Parolini, M. Verani \\ Dipartimento di Matematica, Politecnico di Milano, Milan, Italy \\ *Email address for correspondence: domenico.brunetto@polimi.it \\ Communicated by Elena De Angelis \\ Received on 10 11, 2016. Accepted on 04 10, 2018.
}

\begin{abstract}
This paper aims at bridging Mathematical Modelling and Mathematics Education by studying the opinion dynamics of students who work in small groups during mathematics classrooms. In particular, we propose a model which hinges upon the pioneering work of Hegselmann and Krause on opinion dynamics and integrates recent results of interactionist research in Mathematical Education. More precisely, the proposed model incorporates the following features: 1) the feelings of each student towards the classmates (building upon the so-called "I can" - "you can" framework); 2) the different levels of preparation of the students; 3 ) the presence of the teacher, who may or may not intervene to drive the students towards the correct solution of the problem. Several numerical experiments are presented to assess the capability of the model in reproducing typical realistic scenarios.
\end{abstract}

Keywords: opinion making, bounded confidence, HK model, teacher control

AMS subject classification: 92D25,97C70

\section{Introduction}

In the last decades, within Mathematics Education it has become widely acknowledged that social interactions play a crucial role in student's learning: this has represented a shift from an acquisitionist perspective (according to which the individual's brain is filled by the teacher with knowledge), towards a participationist perspective, where the student engages in mathematical activities with peers and acquires knowledge by interacting with them [1] According to this perspective, each mathematical activity has its roots in our cultural heritage and can be shaped and re-shaped by the group of practitioners. It is within this framework that thinking is conceptualized as a case of communication, since interactionist research postulates the inherently social origin of all human activities [1]. Sfard [1, p. 27] points out that "communication may be defined as a person's attempt to make an interlocutor act, think or feel according to her intentions". Following this view, thinking is thus subordinated to and informed by the demand of making communication effective.

Group work activities in classroom have gained more and more attention in the recent years, since during such activities the students act, interact and communicate much more than in usual frontal lessons settings $[1,2]$. It is well acknowledged that, when students interact in a group, the interaction has not a purely cognitive nature, but it is shaped by affective factors such as emotions, values, wills, attitudes and so on (see, e.g., [3] and [4]). In recent years, many researches have provided evidence that student's attitudes towards mathematics, the classmates and oneself are crucial for the learning process, in particular the feelings "I sense", "I like" and "I can" [4,5]. On these premises, the "I can" - "you can" framework has been proposed [6,7] and it is within this paradigm that this work will propose a new student opinion dynamics model.

Among the extensive studies of human behaviours and interactions, a field of investigation that has gained the attention of researcher in mathematics, physics and computer science (see, e.g., [8,9] and the references therein), opinion dynamics models seems promising and particularly appropriate for our scope. With the term "opinion dynamics" researchers describe a wide class of models for several different social phenomena, such as collective decision making, emergence of ideas, influence of social network on people's opinion and behaviour [10]. Opinion dynamics dates back to French, who defines it as "a way by which 
many complex phenomena about groups can be deduced from a few simple postulates about interpersonal relations" [11, p. 1]. Among the many models that have been proposed since then, the most successful is the bounded confidence model introduced by Hegselmann and Krause in 2002 [12]: according to this model, the evolution of the state of an agent (i.e., the opinion) depends on interactions among the other agents taking place in a bounded domain of confidence.

More recently, this class of models has been applied to a wide range of different fields, ranging from engineering to the life and social sciences, yielding new theoretical and experimental achievements (see, e.g, [13-15] and [16], respectively). Furthermore, very recently a flourishing research activity has focused on the problem of controlling or influencing the opinion dynamics $[9,17-20]$ in order to reach, for instance, the consenus among agents.

However, despite the massive research activity of the last years, to the best our knowledge, there are no mathematical models addressing small group-dynamics in educational context, such as students who are asked to solve a mathematical task working in a group. This paper aims at contributing to fill this gap.

In the construction of our model, in addition to the interaction among students, we take into account also the role of the teacher. This is in agreement with Radford [21] who assigns a central role to the teacher, since she is the only one who knows where the activity should go. The teacher can intervene or not during a small group activity, and her intervention (if any) can have either a mathematical/cognitive or an affective/social nature (see also [22]). For example, a teacher may notice that a student is remaining silent for a long time, hence she can take the pen from the student who is leading the activity and give it to the silent student. This intervention has a social nature. Or, the teacher can comment on a part of a solution the students are discussing. Research fundings in mathematics education [23], in fact, have provided evidence that a teacher in her classroom is exposed to a continuous readjustment process, and the success of her teaching depends on how quickly and how accurately she is able to read the situation. In other words, a teacher needs to make many in-the-moment decisions (see also [24]), on the basis of her ability to read a situation in a specific moment. In our extensive and intensive observation of group interactions [25], in fact, we have observed many secondary school teachers interventions during their students' groupwork activities: what is peculiar is the fact that sometimes the teacher's intervention had been positively received by the students, while some other times it had been completely ignored, apparently without an evident reason. In the mathematical model presented here teacher is considered as an agent, with features that are different from the students' ones, and she may act differently from the students (for example, a teacher may enact a strategy while the students can be considered more naive in the interaction). A similar approach has been taken by $[9,10,18]$, considering agents who take the role of "opinion leaders" modelled through a control variable to achieve consensus. As we will show later, we use the same approach (control variable) but with different aims, namely not to reach a consensus but to reach the correct mathematical solution.

The mathematical model proposed in this paper is based on the framework introduced in [5] and assumes that the acceptance or the refusal of the comments coming from the teacher (as well as the acceptance/refusal of the intervention in the group activity) depends both on the perceived competence that each student of the group has about himself in that particular moment, and the competence that he recognises to his classmates and to the teacher in that particular moment. Another interesting feature of students' interactions is that the correct mathematical solution may be somehow outside the set of the students' initial opinions, therefore it is necessary to develop a mathematical model that takes into account the (not so rare!) possibility to reach the correct solution during the interaction.

The outline of the paper is as follows. In Section 2 we introduce the so-called "I can" - "you can" framework in order to single out the variables that are used to describe the feelings of the students; then in Section 3 hinging upon the above framework, we present the mathematical model describing the dynamics of student opinion. In Section 4 we explore the capability of the model in reproducing realistic scenarios, while in Section 5 we draw some conclusions and we discuss possible future extensions of the present work. 


\section{Codifying the students' feelings}

During any group work activities, the feelings of the students play a crucial role. This is also true in mathematical activities such as problem solving [26]. In particular, the so-called "I can" and "you can" feelings drive and shape both social interactions and mathematical understanding [6]. For example, "I can't" may induce a student to remain silent for a long time, but the stimulus coming from one of his classmates (this latter driven by a "you can" ) may prompt her to intervene. This situation has a social nature. Alternately, the same "I can't" feeling may induce the same student to comment on a part of the mathematical solution she is not grasping, thus seeking for clarification. This latter situation has a mathematical nature. More precisely, the "I can" feeling is the perceived competence of oneself, it identifies how a student feels able to understand a particular mathematical task. On the other hand, we say that the "you can" feeling of a student represents the perceived competence towards his classmate. Hence, the "you can" identifies how much a student is willing to trust and listen to each of his classmates.

In the "I can" - "you can" framework, a student is characterized by two variables, which assume values ranging within $[0,1]$, and the situation can be represented on a coordinate system called the "I can" - "you can" diagram as shown in Figure 1. Four different profiles, that will be referred to as "traits", can be identified:

Trait I Cooperative student is characterized by "I can" close to 1 and "you can" close to 1; this student is willing to share knowledge and mediate his opinion listening to his classmates.

Trait II Obstinate student has "I can" close to 1 but "you can" close to zero, namely he is characterized by a "you can't" attitude ; this student is not willing to listen to other classmates whilst at the same time he wants his classmates to listen to him.

Trait III Isolated student is featured by "I can't" and "you can't" feelings: he does not want to take part in the group activity and in the discussion.

Trait IV Follower student is willing to listen to other classmates but he does not trust himself: a "you can" close to 1 coupled with low self-confidence ("I can't") characterize this profile.

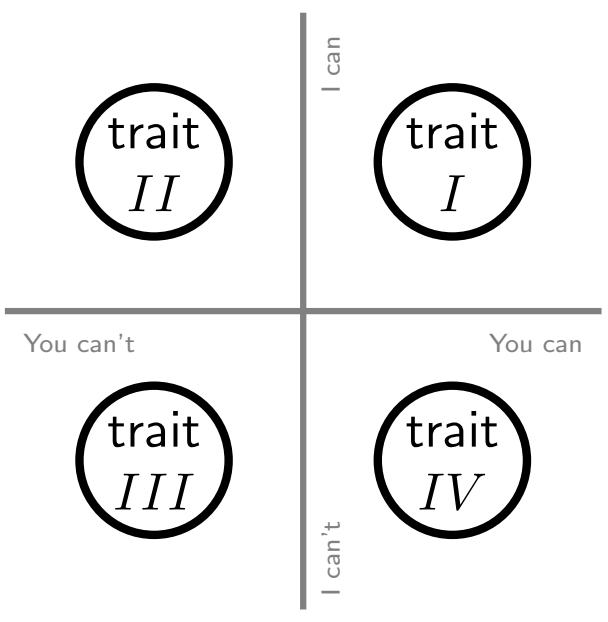

Figure 1. Schema of student traits on the "I can" - "you can" diagram.

We would like to remark that, in order to take into account the many nuances of each student's feeling, it is realistic to consider the "I can" and "you can" variables as continuous. For future reference, we introduce a matrix $K$ collecting the traits of the students. More specifically, let us consider $N$ students and, for $i, j=1, \ldots, N$, let $k_{i j}$ denote the level of competence of the student $j$ perceived by the student $i$, that is the "you can" of the student $i$ towards the student $j$, while $k_{i i}$ indicates the level of "I can" of the student $i$. The matrix

$$
K \in \mathbb{R}^{N \times N}, \quad K=\left[k_{i j}\right]_{i, j=1}^{N}
$$


will be referred to as the trait matrix. For the sake of clarity, we now present a simple example.

Example 2.1. Let us consider $N=3$ students. The following matrix

$$
K=\left[\begin{array}{ccc}
0.9 & 0.2 & 0.15 \\
0.18 & 0.97 & 0.89 \\
0.95 & 0.08 & 0.21
\end{array}\right]
$$

encodes the following traits of the students:

- Student 1 has high level of "I can" $\left(k_{1,1}=0.9\right)$ and low level of "you can" towards both his classmates $\left(k_{1,2}=0.2, k_{1,3}=0.15\right)$; hence he can be classified as obstinate.

- Student 2 has mixed traits: he is quite cooperative with Student $3\left(k_{2,3}=0.89\right)$ whilst he is almost obstinate with Student $1\left(k_{2,1}=0.18\right)$. Moreover, since his level of "I can" is high $\left(k_{2,2}=0.97\right)$ he wants his classmates to listen to him.

- Student 3 has low level of "I can" $\left(k_{3,3}=0.21\right)$ and he is willing to listen to Student $1\left(k_{3,1}=0.95\right)$, but he does not want to interact with Student 2.

\section{The student opinion dynamics model}

In this section, building upon the "I can" - "you can" framework discussed in Section 2, we describe a multi-agent model, based on the concept of bounded confidence introduced in [12], which describes the opinion dynamics of a group of students working together during a classroom activity. The general form of the model is rather standard and reads as follows. Let $N$ denote the number of involved students whose opinion is represented by $x_{i} \in[0,1], i=1, \ldots, N$, then we assume that for each student $i$ his opinion evolves according to the following ordinary differential equation:

$$
\dot{x}_{i}(t)=\sum_{j=1}^{N} w_{i j}(t)\left(x_{j}(t)-x_{i}(t)\right), \quad x_{i}(0)=x_{i}^{0},
$$

where the initial opinion $x_{i}^{0} \in[0,1]$ and $w_{i j}(t)$ are suitable (time dependent) weights measuring how much the student $i$ takes into account the opinion of the student $j$. The corresponding matrix

$$
W(t)=\left[w_{i j}(t)\right]_{i, j=1}^{N}
$$

is named confidence matrix.

The construction of the confidence matrix is crucial and it represents the main contribution of this paper. Indeed a careful construction of the matrix $W$ enables to encode the behaviour of the student $i$ towards the student $j$ in terms of listening, sharing, talking, and mediating. It is clear that such behaviours depend on the student attitudes, therefore it is expected that the trait matrix $K$ introduced in the previous section will enter in the construction of the confidence matrix. This will be addressed in the next section.

Remark 3.1 (On the meaning of the opinion $x_{i}$ ). In this work the opinion $x_{i}$ is the solution that student $i$ has in mind for the proposed task. For sake of simplicity, it is represented by a scalar value in the range $[0,1] \subset \mathbb{R}$. More involved characterizations of the student opinion (for instance, a vector valued $\mathbf{x}_{i} \in \mathbb{R}^{d}$ describing the different steps of a solution strategy) could be also considered to account for more complex situations. However, this goes beyond the scope of the present work.

\subsection{Construction of the confidence matrix}

In this section we will build $W(t)$ as a time dependent matrix with elements depending on the opinions $x_{i}(t), i=1, \ldots, N$. The construction will be done in several steps. 
First, we introduce the rough weight $w_{i j}^{*}(t)$ defined as the product of the attitude score $a_{i j}$ and the perceived distance score $\varphi_{i j}(t)$ :

$$
w_{i j}^{*}(t)=a_{i j} \cdot \varphi_{i j}(t) .
$$

The element $w_{i j}(t)$ is then built as normalization of the rough weights $w_{i j}^{*}(t)$, i.e.

$$
w_{i j}(t)=\frac{w_{i j}^{*}(t)}{\sum_{j=1}^{N} w_{i j}^{*}(t)}, \forall i=1, \ldots, N .
$$

The score $a_{i j}$ encodes the attitude of the student $i$ towards the student $j$ and it is defined as

$$
a_{i j}=k_{i j}+0.1
$$

where $k_{i j}$ is an element of the trait matrix $K$. Hence, the more the student $i$ feels that the student $j$ is reliable, the more the opinion of the latter will influence the opinion of the former. The presence of the shift term 0.1 introduces a small level of interaction even when student $i$ exhibits a high level of "I can't" towards student $j\left(k_{i j}=0\right)$.

The perceived distance score $\varphi_{i j}(t)$ modulates the opinion distance $d_{i j}(t)=x_{j}(t)-x_{i}(t)$ according to the reciprocal attitudes of students $i$ and $j$. Thus, we propose to define the perceived distance score as:

$$
\varphi_{i j}(t)=\beta_{i j}\left(\psi_{j}\left(\left|d_{i j}(t)\right|\right)\right),
$$

where $\beta_{i j}$ is the confidence function:

$$
\beta_{i j}(z)= \begin{cases}1-\frac{1}{\varepsilon_{i j}}|z|, & |z| \leq \varepsilon_{i j} \\ 0, & \text { otherwise }\end{cases}
$$

and $\psi_{j}$ is the perceived distance function:

$$
\psi_{j}(z)=z \cdot 2^{-k_{j j}}
$$

The perceived distance function $\psi_{j}$ has been designed so that a student with a high value $k_{j j}$ of "I can" is able to get his classmates to listen. Roughly speaking, a student with high level of "I can" is able to reduce the opinion distance between himself and his classmates.

The confidence function $\beta_{i j}$ is a simple variant of the confidence interval introduced by Hegenselman and Krause, and it allows to reproduce the typical dynamics observed in real situations where student $i$ is willing to listen only to students with "close" opinions. This is obtained by introducing suitable student dependent threshold $\varepsilon_{i j}$, which is highly influenced by the trait of student $i$ :

$$
\varepsilon_{i j}=\left[\varepsilon_{2}+\left(k_{i i}-1\right)\left(\varepsilon_{2}-\varepsilon_{3}\right)\right]\left(1-k_{i j}\right)+\left[\varepsilon_{1}+\left(k_{i i}-1\right)\left(\varepsilon_{1}-\varepsilon_{4}\right)\right] k_{i j},
$$

where $\varepsilon_{l}$, with $l=1,2,3,4$, are thresholds parameters associated to the four traits discussed in the previous section.

Remark 3.2 (On the choice of $\varepsilon_{l}$ ). The parameters $\varepsilon_{l}$ have been chosen so that the solution of (2) qualitatively reproduces realistic student work group activities. High threshold values $\left(\varepsilon_{4}=0.7\right.$ and $\varepsilon_{1}=$ 0.5) have been employed for students with high level of "you can" (trait I and IV) since they are open to listen to students who have relatively different opinions from theirs. However, the cooperative student (trait I), who has a high level of "I can", is less inclined to listen than the follower student (trait IV).

Low threshold values $\left(\varepsilon_{2}=0\right.$ and $\left.\varepsilon_{3}=0.05\right)$ are used for the other two types of student: the obstinate student (trait II) does not listen to other opinion, whilst the isolated student (trait III) might change his opinion only if he listens to an opinion very close to his own. 
Example 3.1. Let us compute the confidence matrix for a simple case where only two students interact. The first student is cooperative (trait I) while the second one is a follower (trait IV). Their initial opinions are $x_{1}^{0}=1$ and $x_{2}^{0}=0$, respectively, and the correspondent attitude matrix $K$ is given by:

$$
K=\left[\begin{array}{ll}
1 & 1 \\
1 & 0
\end{array}\right]
$$

Using the attitude score (5), the perceived distance score (6) and the threshold values introduced in Remark 3.2 , the confidence matrix for this simple case is given by

$$
W(0)=\left[\begin{array}{cc}
1 & 0 \\
\frac{22}{29} & \frac{7}{29}
\end{array}\right]
$$

Note that matrix $W(0)$ is not symmetric, due to the different traits of the students. The resulting dynamical system (2) is numerically solved using an adaptive Runge-Kutta method (ode23 solver of Matlab(C) and the evolution of the opinions is displayed in Figure 2. Initially, Student 2 (line 2) moves towards Student 1 (line 1), while the latter does not change his opinion until Student 2 is enough close to him. Since then, the weights $w_{1,2}(t)$ becomes not null and also Student 1 starts to mediate his opinion moving slowly towards Student 2 . Finally, they reach a consensus, namely an agreement about the solution (right or not) to the task.

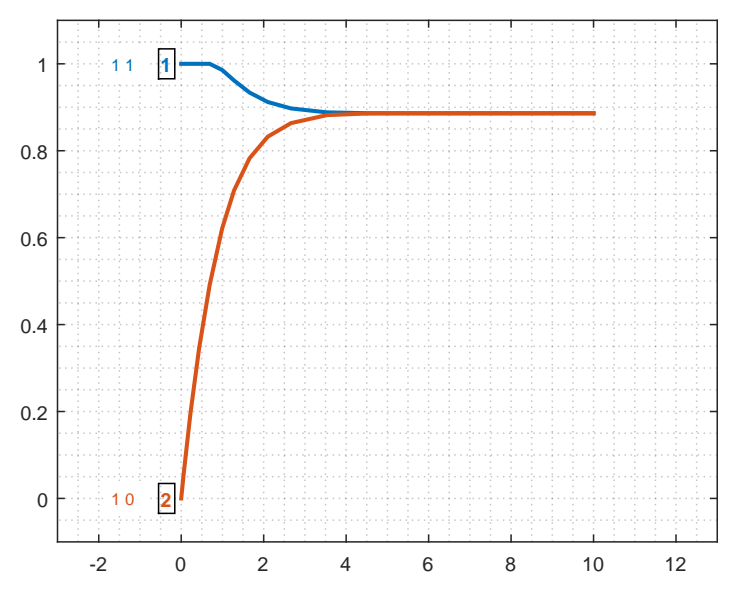

Figure 2. Example of 2-student opinion dynamics. Student 1 (line 1) is cooperative while Student 2 (line 2) is follower.

\subsection{The role of the correct solution}

It is worth noting that at each time the model (2) predicts a distribution of opinions that belong to the convex hull of the initial opinions. Thus, the opinions at the final time $t=T$ will range within the extreme opinions at the initial time $t=0$. This is in general acceptable if one has in mind, for example, a group of experts judging a project (in this case the "correct opinion" does not exist!). However, such a restriction on the range of the final opinions is not acceptable in our context, since the students may reach a consensus on a wrong answer or on an incorrect solution to the problem. During a group work activity the students are typically challenged to find the correct answer to a given exercise and sometimes the correct solution to the task does not belong to the convex hull of the initial opinions. However, it may happen in practice that the interactions between students contribute to reach a completely "new" opinion (i.e. not belonging to the convex hull of the initial opinions). For example, this is the case when two students with different, partial but complementary, competences work together. 
In view of the above discussion, we add an extra "agent" who plays the role of the correct solution. More precisely, the correct solution acts as an agent who never changes his opinion (i.e., the correct answer to the task), $\dot{x}_{0}(t)=0$ and $w_{00}(t)=1$. In addition, this extra agent is able to strongly attract a certain class of students, namely those who have studied and are confident with the possibility of solving the given problem. We assume that students with low mathematical knowledge are (at least at the beginning of the interaction) far from the correct solution, as well as they are weakly attracted by it. We understand "mathematical knowledge" as a mix of "level of study" and "perception of understanding". In order to introduce the variable "mathematical knowledge" into the model, the trait matrix $K$ is extended by adding an extra row $k_{0 i}$ which represents the level of study of the student $i$ and an extra column $k_{i 0}$ which represents the level of perception of understanding of the student $i$. Accordingly, we define the extended trait matrix $\mathcal{K}$ which considers the student traits and the correct solution, as follows

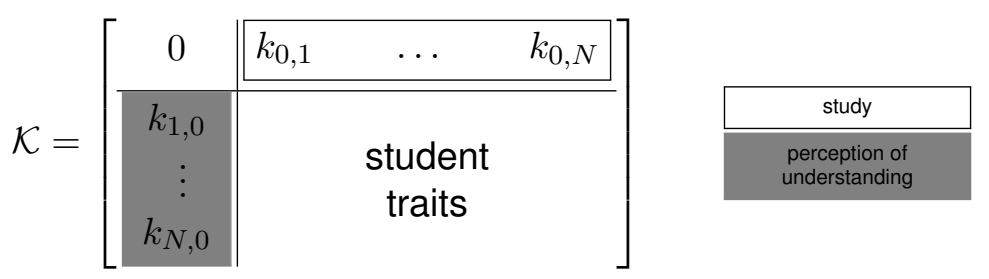

Being an extension of the matrix $K$, we interpret $k_{0, i}$ as the level of study because, in a sense, it represents the "you can" of mathematics towards the student $i$. Analogously, the element $k_{i, 0}$ is viewed as the "you can" of the student $i$ towards the mathematics. The latter can be seen also as a student's computational skills, his perseverance in the task, his appreciation of mathematics.

The weight $w_{i 0}$ encoding the interaction between the student $i$ and the correct solution is defined as

$$
w_{i 0}=c_{1}\left(k_{0 i} k_{i 0}+c_{0} k_{0 i}\left(1-k_{i 0}\right)\right)
$$

with $c_{0}>0$ and $0<c_{1} \leq 1$. A convenient choice made in our simulations is $c_{0}=\frac{1}{10}, c_{1}=\frac{1}{2}$. The heuristic behind equation (12) is that a student who has studied and has strong perception of understanding is attracted by the correct solution, while a student who has studied but has low perception of understanding is very weakly attracted by the correct solution.

In summary, the opinion dynamics of the student $i$ is given by

$$
\dot{x}_{i}(t)=\sum_{j=0}^{N} w_{i j}(t)\left(x_{j}(t)-x_{i}(t)\right), \quad i=1, \ldots, N
$$

where $x_{0}(t)=x_{0}$ for any $t$. Finally, the rough weights $w_{i j}^{*}$ with $j=1, \ldots, N$ are normalized to obtain the final weights $w_{i j}$ such that

$$
\sum_{j=1}^{N} w_{i j}=1-w_{i 0}
$$

while the weight $w_{i 0}$, defined by Equation (12), is not rescaled.

\subsection{The teacher as a leader}

A crucial aspect in describing student's opinion dynamics is the presence of the teacher, who plays an important role as she knows both the correct solution and the procedure(s) that the students should follow to get it. Therefore, we introduce the teacher as a new agent with opinion $x_{N+1}$ who influences the opinions of the students. More precisely, $x_{N+1}$ evolves according to a strategy. For example a strategy may consist of staying silent until a certain moment, then interacting by providing hints. In this work, we consider the teacher's strategy as a priori-defined. Adding the contribution of the teacher, the student 
opinion dynamics model becomes

$$
\left\{\begin{array}{l}
x_{0}(t)=x_{0} \\
\dot{x}_{i}(t)=\sum_{j=0}^{N+1} w_{i j}(t)\left(x_{j}(t)-x_{i}(t)\right), \quad i=1, \ldots, N \\
\dot{x}_{N+1}(t)=u(t)
\end{array}\right.
$$

where $u(t): \mathbb{R}_{0}^{+} \rightarrow \mathbb{R}$ represents the evolution of teacher's opinion. Roughly speaking, $u(t)$ represents the strategy implemented by the teacher. The weight $w_{i, N+1}$, which encodes the attitude of the student $i$ towards the teacher, is computed according to the general procedure described above; this means that the teacher can be listened or not by a student according to the value of $k_{i, N+1}$ of the trait matrix $K$. The traits are now collected in the further extended trait matrix $\mathcal{K}$ which now assumes the following form:

$\mathcal{K}=\left[\begin{array}{c|ccc|c}0 & k_{0,1} & \cdots & k_{0, N} & 0 \\ \hline k_{1,0} & & & & \\ \vdots & & \text { student } & & k_{1, N+1} \\ k_{N, 0} & & \text { traits } & & \vdots \\ \hline 0 & 0 & \cdots & 0 & 1\end{array}\right]$

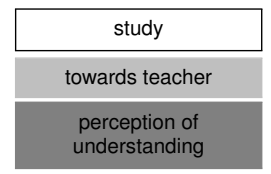

\section{Numerical results}

This section reports two sets of numerical results dealing with the opinion dynamics of a group of 3 students. Each set includes the dynamics with or without the influence of the correct solution, and, eventually, with the interventions of the teacher. These dynamics are different due to the different traits of the students involved.

Test case 1: cooperative students at work. Figure 3(a) shows an example of 3 cooperative students, with low mathematical knowledge, who share and mediate their initial opinion reaching a consensus, that is they reach an agreement about the solution of the task even if it is not the correct one. Since the students have not studied (thus the counterpart in the mathematical model is that the agent "correct solution" has no effect) and they are cooperative, they are willing to listen to each other and mediate their opinions, but they move away from the correct solution. Figure 3(b) reports the dynamics in which the correct solution plays an active role. In particular, the trait matrix $K$ remains the same, but in this case Student 3 has studied $\left(k_{0,3}=1\right)$ even if he typically makes mathematical mistakes $\left(k_{3,0}=0.5\right.$, namely low perception of understanding), while the other two students have not studied $\left(k_{0,1}=k_{0,2}=0\right)$ but they have good perception of understanding $\left(k_{1,0}=k_{2,0}=0.9\right)$. The dynamics reported in Figure 3(b) can be interpreted as follows: Student 3, who has studied, has an initial opinion close to the correct solution, then he moves towards the classmates explaining them the topic of the task and how to solve it. Finally, followed by the other two students, he goes towards the solution but slowly because of his difficulty in making computations. In Figure 4 we explore the influence of the teacher, considering the same students' situation of Figure 3(b). In the dynamics reported in Figure 4(a), the teacher, from the beginning, takes part in the discussion providing hints for the correct solution. Note that all students listen to the teacher $\left(k_{i, 4}=1, i=1,2,3\right)$ and her opinion is constant in time, namely $u(t)=0$ in Equation (14).

In principle, the teacher acts to improve the work group discussion allowing students to give the correct solution, however it is not easy for her to identify an effective strategy to adopt. In Figures $4(\mathrm{~b})-4(\mathrm{~d})$ we report the dynamics associated to different strategies $(u(t) \neq 0)$ corresponding to different 


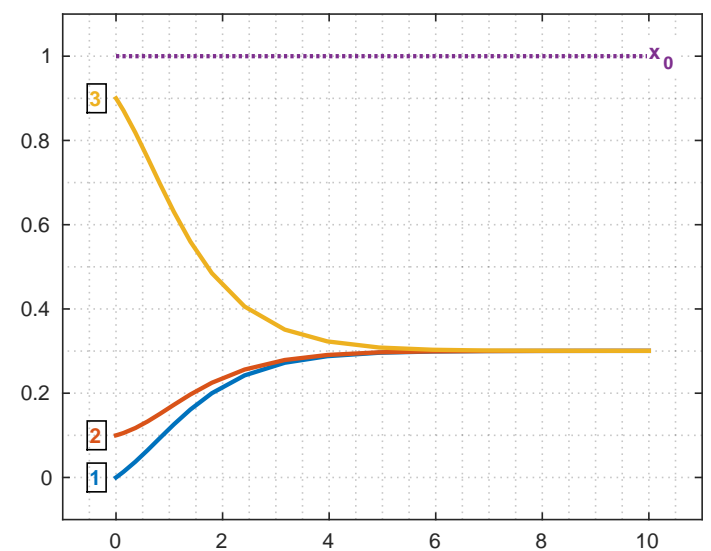

(a)

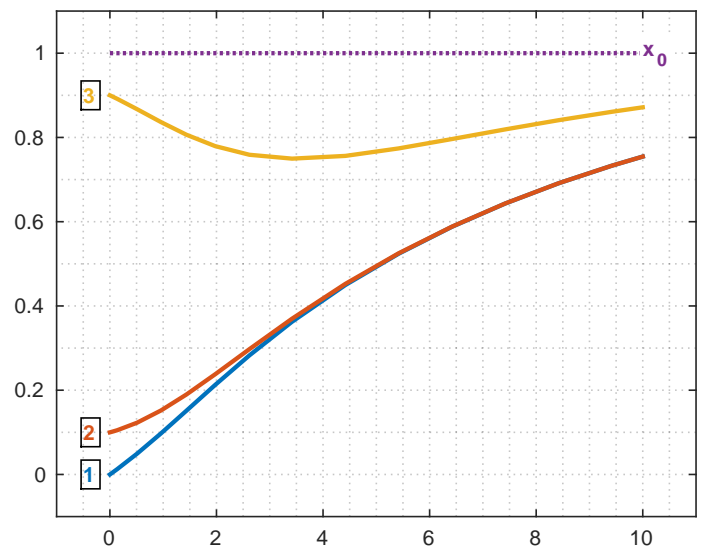

(b)

Figure 3. Test case 1: 3-Student opinion dynamics and the influence of correct solution. The solid lines represent the evolution of the opinions associated to each student. The dotted line, labelled by $x_{0}$, identifies the correct solution.

choices for the function $u(t)$, namely

$$
\begin{aligned}
& u_{b}(t)=\frac{2}{25}(t-5), \\
& u_{c}(t)=\left\{\begin{array}{ll}
-1, & t \in[0 ; 1] \\
\frac{1}{9}, & t \in(1 ; 10]
\end{array},\right. \\
& u_{d}(t)= \begin{cases}-\frac{1}{2}, & t \in[0 ; 1 / 3] \\
0, & t \in(1 / 3 ; 7] \\
\frac{1}{20}, & t \in(7 ; 10]\end{cases}
\end{aligned}
$$

where the subscripts correspond to the sub-figure in Figure 4(a)-4(d), respectively.

Employing the strategy $u_{b}$ the teacher ranges all the possible opinions in the time $[0,10]$, starting from the correct solution $(x=1)$, reaching $x=0$ at $t=5$ and going back to the correct solution. The aim of this strategy is to intercept the students and drive them to the correct solution. However, this strategy is not completely effective because of the velocity of the evolution. Indeed, the teacher rushes to achieve her goal and the students do not manage to take fully advantage of her hints. Adopting the strategy $u_{c}$ the teacher wants to reach the "worst" students, namely Student 1 and Student 2 who are far from the correct solution, and drive them towards it. If compared with strategy $u_{b}$, strategy $u_{c}$ allows to drive students 1 and Student 2 closer to the correct solution. However we note that Student 3 is driven far from the correct solution, with respect to his initial opinion. Finally, employing the strategy $u_{d}$ the teacher moves closer to Student 3 and somehow endorses him forming a "pole", which attracts the other two students. When all the opinions are clustered $(t=7)$, the teacher moves towards $x_{0}$ bringing the students closer to the correct solution.

As it is clear from the above discussion, it is not easy to define an a priori optimal strategy that ensures a desired dynamics. Thus it becomes crucial to identify systematic ways to design optimal interventions.

Test case 2: Obstinate controls the dynamics. In this set of simulations a heterogeneous group of student is considered. The trait matrix is reported in (19), Student 1 is obstinate, Student 2 is a follower and Student 3 has a mixed trait between follower and cooperative, he follows Student 2 and he is cooperative with Student 1. Details are reported in the following traits matrix:

$$
K=\left[\begin{array}{ccc}
1 & 0 & 0 \\
1 & 0 & 0.5 \\
0.5 & 1 & 0.5
\end{array}\right] \text {. }
$$




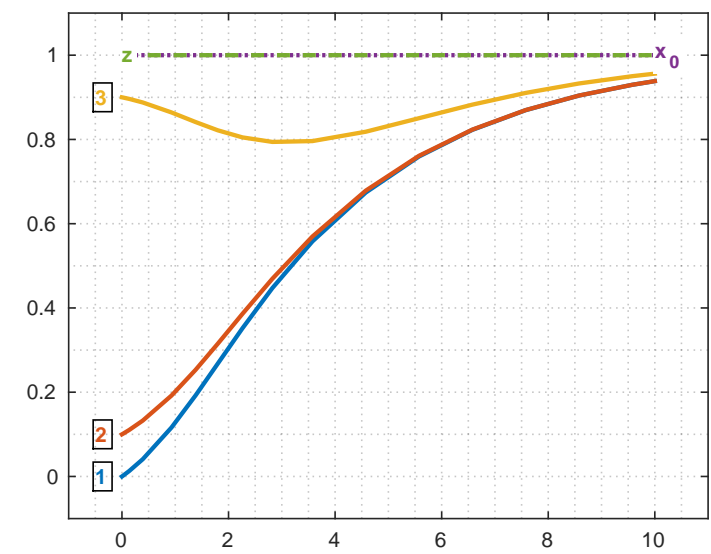

(a)

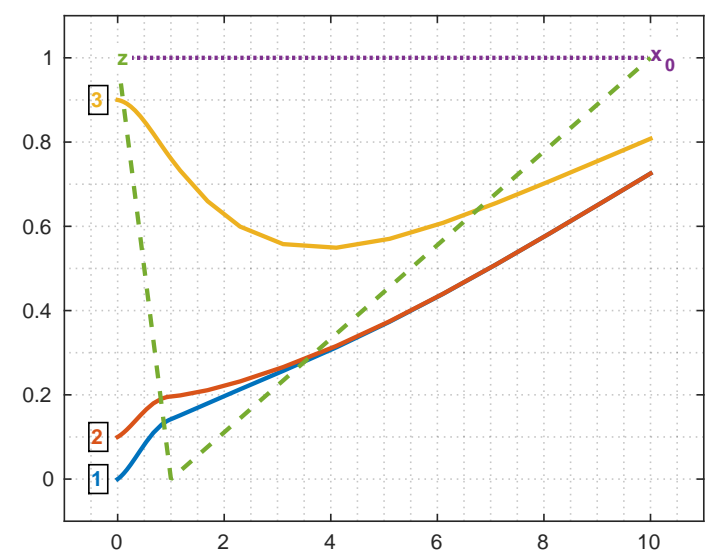

(c)

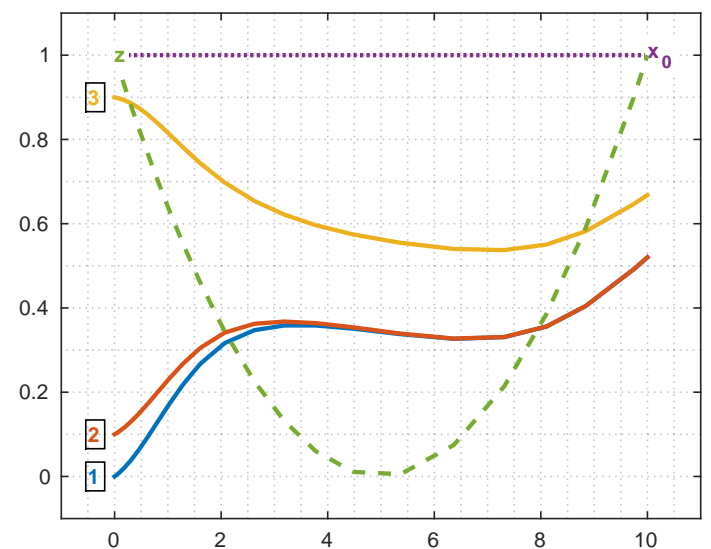

(b)

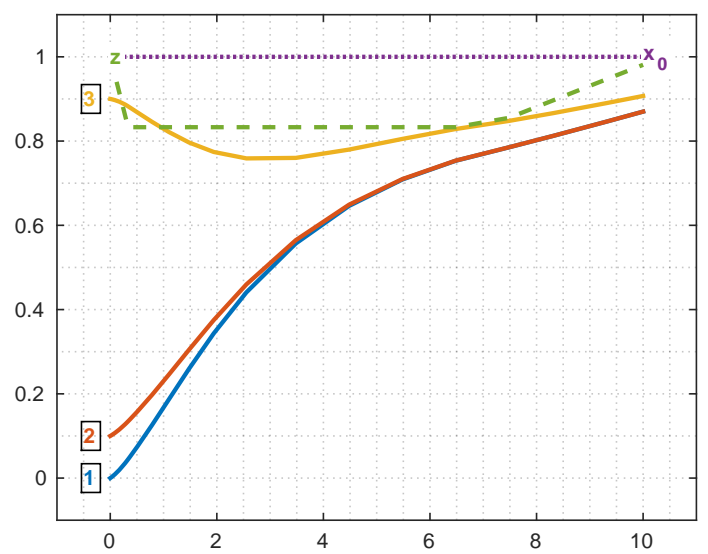

(d)

Figure 4. Test case 1: 3-Student opinion dynamics with different strategies for the teacher intervention. The dashed green line represents the evolution of the teacher $\left(x_{N+1}\right)$ opinion.

The resulting dynamics is showed in Figure 5: Student 1, who does not change his opinion (blue line), monopolizes the dynamics by attracting the other students since they present a low level of "I can" together with a pronounced tendency to trusting other classmates; Student 2, who trusts more Student $1\left(k_{2,1}=1\right)$ than Student $3\left(k_{2,3}=0.5\right)$, moves quickly towards the opinion of Student 1 and slowly towards the one of Student 3. Finally, Student 3 cooperates with the other two classmates reaching their opinion rather quickly. Once Student 2 and Student 3 are close to each other and to Student 1, they follow this latter reaching a consensus. It is worth noting that Student 3 was initially close to the correct solution, but, because of his attitude, ends very far from it. Finally, we remark that the correct solution has no effect since none of them has studied. On the contrary, when mathematical knowledge is considered in the dynamics, different behaviours and results are obtained, as shown in Figure 6. In this case, we assume that Student 1 has moderately studied $\left(k_{0,1}=0.5\right)$ and is characterized by a perception of understanding of $k_{1,0}=0.5$, while the other two students have not studied at all $\left(k_{0,2}=k_{0,3}=0\right)$ but they have very high perception of understanding $\left(k_{2,0}=k_{3,0}=1\right)$. There follows that Student 1 drives his classmates towards the correct solution, even if they do not reach it because of lack of time, see Figure 6(a). Figure 6(b) shows the dynamics in case Student 3 is the only one who has studied. In this case, where $k_{0,3}=1, k_{3,0}=0.5$ and other traits relative to the mathematical attitudes are null, the knowledge and the mathematical skills of Student 3 are confused by the eloquence of Student 1, who somehow controls the dynamics, whilst Student 2 is between two "poles" represented by the obstinate Student 1 and Student 3 with low self-confidence. As a consequence, the work group activity ends with three very different opinions, in other words there is no clue what could be the correct answer to the 


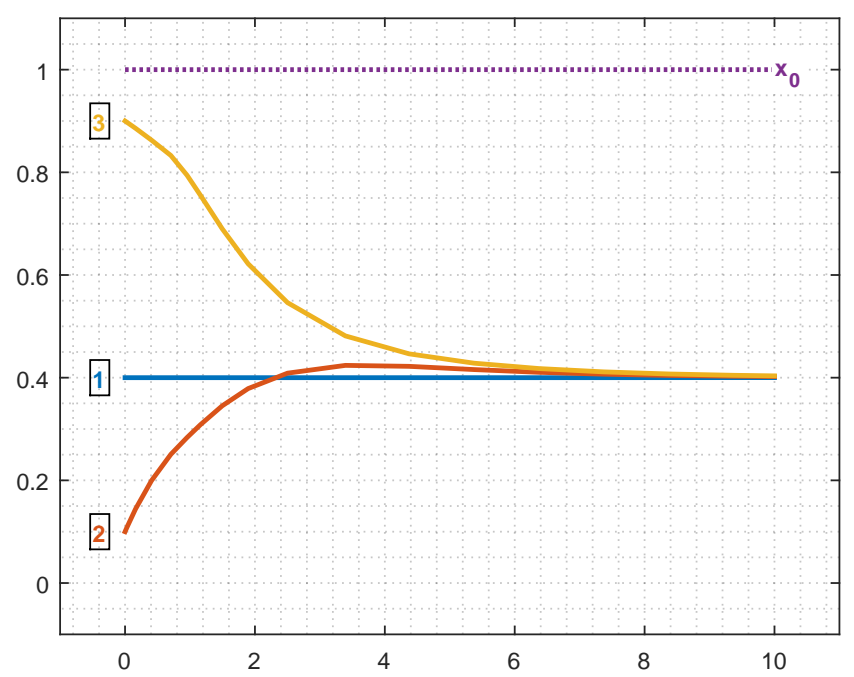

Figure 5. Test case 2: 3-Student opinion dynamics (of heterogenous group of students). Solid lines represent student opinion while the dotted one represents the correct solution, which has no effect on students in this case. Student 1 is obstinate, while students 2 and 3 are almost cooperative.

given task. When the dynamics is getting more complex and the answer to the task could not be achieved

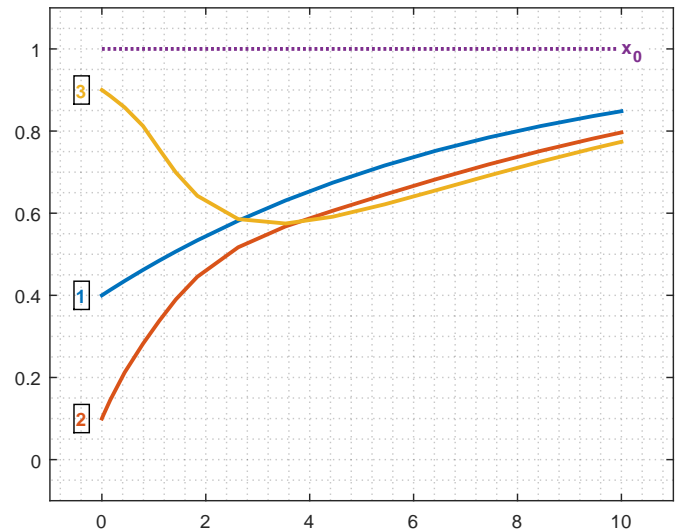

(a)

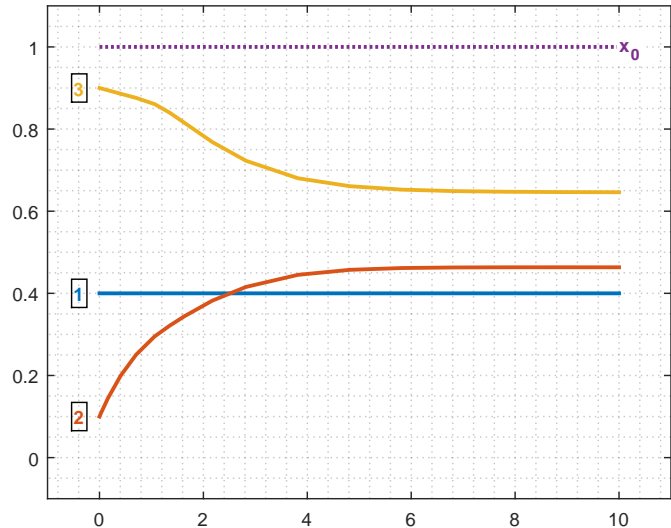

(b)

Figure 6. Test case 2: 3-Student opinion dynamics (of heterogenous group of students). Student 1 is cooperative while students 2 and 3 are almost cooperative. Two different settings: (a) Student 1 has studied; (b) Student 3 has studied.

by the students left alone during the group work activity, the role of the teacher becomes really crucial. Figure 7 reports different strategies activated by the teacher to drive the students towards the correct solution in a priori fixed amount of time. The extended trait matrix used in these simulations is

$$
\mathcal{K}=\left[\begin{array}{ccccc}
0 & 0 & 0 & 0 & 0 \\
0 & 1 & 0 & 0 & 0.5 \\
0 & 1 & 0 & 0.5 & 1 \\
0 & 0.5 & 1 & 0.5 & 1 \\
0 & 0 & 0 & 0 & 1
\end{array}\right]
$$

We recall that the last column of $\mathcal{K}$ is related to the teacher influence. In this example, Student 2 and Student 3 are willing to listen to the teacher $\left(k_{2,4}=k_{3,4}=1\right)$ whilst Student 1, due to his attitude, has some resistance to listen to her. 
In the corresponding dynamics reported in Figure 7 the teacher interacts with the students during the whole discussion using the following different strategies

$$
\begin{aligned}
& u_{a}(t)=0 \\
& u_{b}(t)=\frac{2}{50}(t-5) \\
& u_{c}(t)= \begin{cases}-\frac{3}{5}, & t \in[0 ; 1] \\
\frac{1}{15}, & t \in(1 ; 10]\end{cases} \\
& u_{d}(t)= \begin{cases}-\frac{1}{5}, & t \in[0 ; 1 / 3] \\
0, & t \in(1 / 3 ; 2] \\
\frac{1}{20}, & t \in(2 ; 3] \\
0, & t \in(3 ; 4] \\
\frac{1}{20} & t \in(4 ; 10]\end{cases}
\end{aligned}
$$

where the subscripts of the control $u(t)$ refer to the Figure $7(\mathrm{a})-7(\mathrm{~d})$, respectively.

In Figure 7(a) the teacher's opinion is constant during the activity and she provides hints for the correct solution which are grasped only by Student 3, the closest one, who, in turn, mediates between the hints of the teacher and the opinion of the obstinate student. Furthermore, Student 3 is able to attract Student 2 with the help of the teacher since this latter student trusts both of them. In Figure 7(b), the teacher ranges all the possible opinions getting Student 1 involved as well; however, from time $t=5$ the teacher comes back to the correct solution too quickly loosing the students, namely the complexity of her suggestions increases too much to be fully caught by them. With strategy $u_{c}$ (Figure $7(\mathrm{c})$ ) the

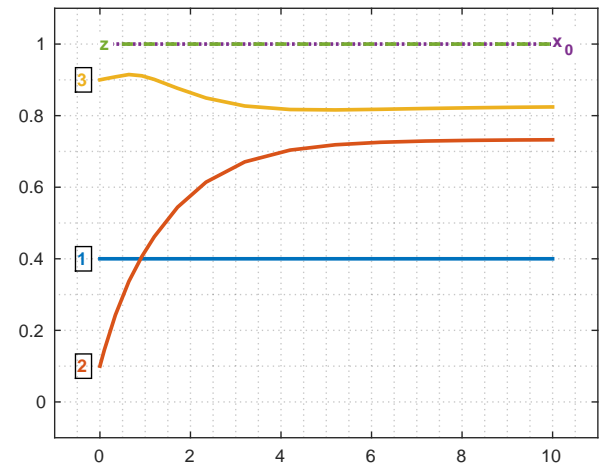

(a)

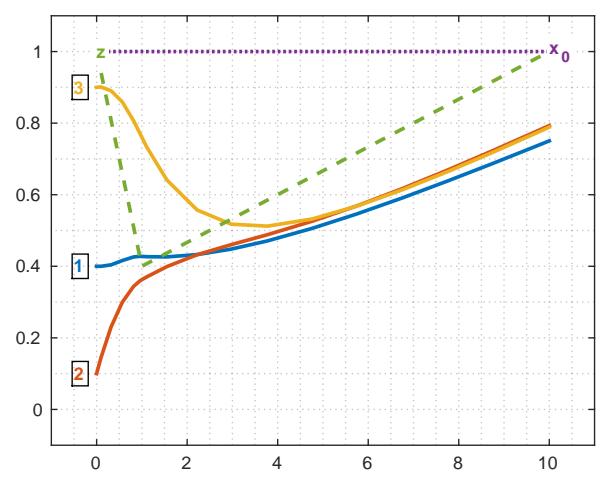

(c)

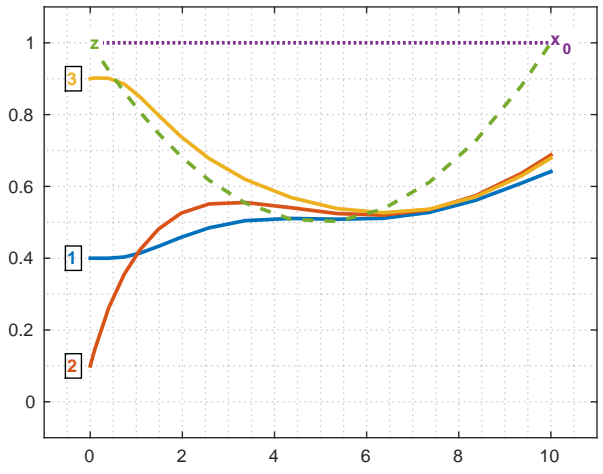

(b)

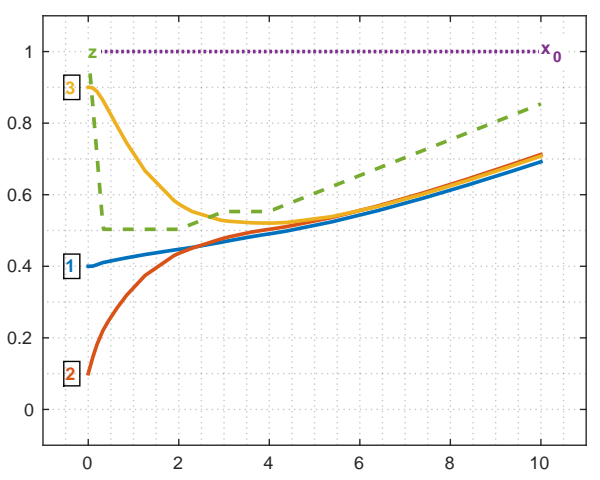

(d)

Figure 7. Test case 2: 3-Student opinion dynamics (of heterogenous group of students). The presence of the dashed green line means teacher intervenes and it represents the evolution of the teacher $\left(x_{N+1}\right)$ opinion.

teacher reaches the obstinate student and convinces him to follow her; however, since the evolution of 
the teacher's opinion is too fast, the students stop following her very early. Similarly, the strategy $u_{d}$ (see Figure 7(d)) allows the teacher to keep the students closer to her, but not enough to drive them towards the solution. Nevertheless, in all the considered dynamics, the teacher succeeds in driving Student 1 and Student 2 closer to the correct solution, while, unfortunately, she fails with Student 3 who moves away from $x_{0}$.

We dwell on Figure 7 to observe that the teacher's strategy $u_{c}$ allows the group of student as a group to get closer to the correct solution, while with the strategy $u_{a}$ only Student 3 get as much closer to the correct solution. To design which one is the best strategy for the teacher depends on the teacher's goal. For example, in the latter case the teacher may want to reduce the gap among the students, or in the other cases the teacher may want the group to work together and reach a common and shared solution.

We remark that the teacher has a further possibility: instead of performing an intervention with a mathematical nature, her intervention can have a social nature. For instance, she can silence students or prompt the silent student to talk. The last dynamics presented in Figure 8, reports an example of this kind of strategy. Firstly, the teacher observes the discussion, at a certain point $(t=2)$ she intervenes on two fronts: transforming the obstinate Student 1 in a follower $\left(k_{1,1}=0, k_{1,4}=1\right)$ and suggesting the correct solution to all students $\left(k_{i, 4}=1\right)$. Since she is confident that Student 1 has understood the task and the resolution strategy, she stops the intervention at time $t=6$ and leaves the dynamics to evolve naturally. In this case $u(t)=0$ and it is the matrix $\mathcal{K}$ that changes in time, because $u(t)$ models the mathematical nature of the teacher's intervention, while a change in $\mathcal{K}$ models the social one. Such a strategy allows the teacher to focus on the obstinate student (Student 1), who may acts as a leader, leaving him the role of driving his classmates towards the solution. Of course, up to a certain extent, the hints of the teacher are taken into account by students 2 and 3 as well. As a consequence, at the end of the dynamics, all students are very close to the correct solution even though they do not completely reach the correct solution $x_{0}$.

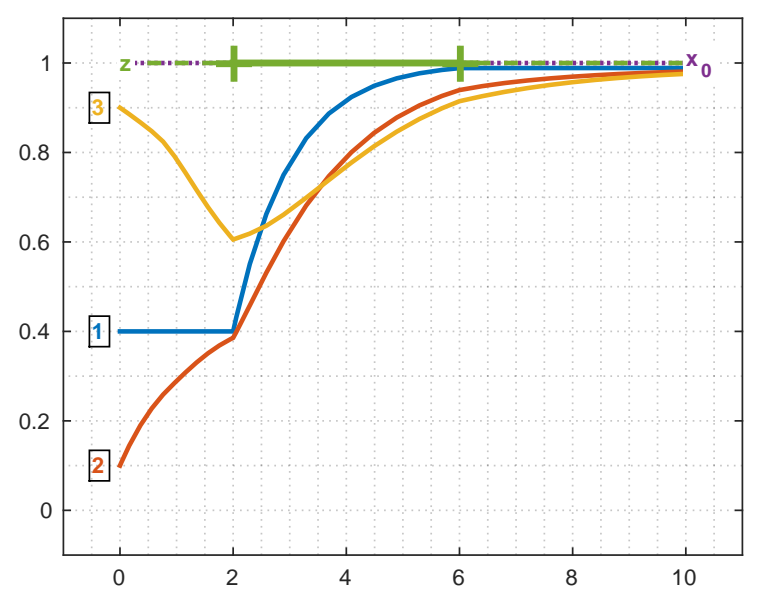

Figure 8. 3-Student opinion dynamics of heterogenous group. Mix intevention strategy. The green solid line represents the teacher hints, while green markers represent the instant where teacher changes the trait of Student 1 (blue line).

If we compare 7 and 8 we can notice that in the latter case all the students get closer to the correct solution with respect to any case depicted in 7 . It seems that the social nature of teacher's interventions is more efficient and this is in line with the interactionist approach, according to which social interactions determine cognitive understanding and not vice versa [1].

\section{Conclusion and future work}

In this paper we introduce and discuss a mathematical model suited for describing the students' opinion dynamics during group work activities at school. Specifically, we assume that, when the interaction among the students starts, each one of them has his "opinion" about the task/problem to be solved. This opinion is modelled as a value ranging from 0 to 1 and each student is assigned a value. Research in 
Mathematics Education have provided evidence, however, that the dynamic of the interaction among students depends also on the affective status of each student. In particular, we take into account the "I can" (self-confidence) and the "you can" (ability recognised to each classmate), and we plug into the basic model the matrix of coefficients so that a student with high "you can" is more likely to listen to his classmates and change his opinion. In order to take into account also the various "levels" of mathematical ability, we introduce a further set of coefficients that model the propensity to be attracted by the correct solution to the problem. The correct solution is modelled as an "agent" in our study, since (like all the students involved) it may attract the students or not. We assume that a student with low mathematical knowledge is less likely to be attracted by the correct solution, with respect to a student with higher mathematical ability. To sum up, our model considers the role of mathematical knowledge (in terms of "correct solution" to a given task), of each student's ability and of affective issues such as self-confidence and trust towards each classmates, in determining the dynamics of a small group of students working on a task at school.

Moreover, the teacher, who acts as an agent, may intervene to improve the group work activity. Our simulations show that, when the teacher's intervention has a social nature, the group may get closer to the correct solution as a group, while when the nature of the teacher's intervention is rather cognitive, only some students seem to benefit from it. The large set of numerical experiments presented shows the capability of the model to reproduce different "realistic scenarios". However, a mathematical understanding of the "optimal" intervention of the teacher represents a challenging development of the present work, that is the definition of a suitable optimal control problem and its numerical solution $[9,18,20]$ which is currently under investigation.

Finally, we would like to study the possible role that improved versions of the present model can play in supporting teachers who want to handle student-centred activities in classroom. In particular, a simulator based on the present model can help teachers to improve their abilities to manage in-the-moment decision making $[27,28]$. We believe that, by allowing prototypisation and generalisation, a simulator of small group activities will serve the purpose of enhancing even more teachers' understanding of group dynamics and hence contribute to the design of mathematics lessons that involve the students as active participants.

\section{References}

1. A. Sfard, There is more to discourse than meets the ears: Looking at thinking as communicating to learn more about mathematical learning, Educational studies in mathematics, vol. 46, no. 1-3, pp. 13-57, 2001.

2. A. H. Schoenfeld, What makes for powerful classrooms, and how can we support teachers in creating them? a story of research and practice, productively intertwined, Educational Researcher, vol. 43, no. 8, pp. 404-412, 2014.

3. M. Hannula, J. Evans, G. Philippou, and R. Zan, Affect in mathematics education-exploring theoretical frameworks. research forum., International Group for the Psychology of Mathematics Education, 2004.

4. P. Di Martino and R. Zan, The construct of attitude in mathematics education, in From beliefs to dynamic affect systems in mathematics education, pp. 51-72, Springer, 2015.

5. C. Andrà and P. Liljedahl, "I sense" and "I can": Framing intuitions in social interactions, in Proceedings of the joint meeting of PME and PMENA, vol. 1, pp. 49-56, Vancouver, CA:PME, 2014.

6. C. Andrà, D. Brunetto, N. Parolini, and M. Verani, 'I can - you can': Cooperation in group activities, in Proceedings of the Ninth Congress of the European Society for Research in Mathematics Education (K. Krainer and N. Vondrová, eds.), pp. 1109-1115, Feb. 2015.

7. C. Andrà, D. Brunetto, N. Parolini, and M. Verani, "Four fundamental modes of participation in mathematics group activities." (to appear).

8. N. Bellomo, Modeling complex living systems. Modeling and Simulation in Science, Engineering 
and Technology, Birkhäuser Boston, Inc., Boston, MA, 2008. A kinetic theory and stochastic game approach.

9. G. Albi, L. Pareschi, G. Toscani, and M. Zanella, Recent advances in opinion modeling: Control and social influence, in Active Particles, Volume 1: Advances in Theory, Models, and Applications (N. Bellomo, P. Degond, and E. Tadmor, eds.), pp. 49-98, Springer International Publishing, 2017.

10. J. Lorenz, Continuous opinion dynamics under bounded confidence: A survey, International Journal of Modern Physics C, vol. 18, no. 12, pp. 1819-1838, 2007.

11. J. R. French Jr, A formal theory of social power., Psychological review, vol. 63, no. 3, p. 181, 1956.

12. R. Hegselmann, U. Krause, et al., Opinion dynamics and bounded confidence models, analysis, and simulation, Journal of Artificial Societies and Social Simulation, vol. 5, no. 3, 2002.

13. V. D. Blondel, J. M. Hendrickx, and J. N. Tsitsiklis, On krause's multi-agent consensus model with state-dependent connectivity, IEEE transactions on Automatic Control, vol. 54, no. 11, pp. 2586-2597, 2009.

14. C. Canuto, F. Fagnani, and P. Tilli, An Eulerian approach to the analysis of Krause's consensus models, SIAM J. Control Optim., vol. 50, no. 1, pp. 243-265, 2012.

15. J. Lorenz, Multidimensional opinion dynamics when confidence changes, Economic Complexity, Aixen-Provence, 2003.

16. J. Lorenz, H. Rauhut, F. Schweitzer, and D. Helbing, How social influence can undermine the wisdom of crowd effect, Proc. of the National Academy of Sciences, vol. 108, no. 22, pp. 9020-9025, 2011.

17. R. Kaur, R. Kumar, A. P. Bhondekar, and P. Kapur, Human opinion dynamics: An inspiration to solve complex optimization problems, Scientific reports, vol. 3, 2013.

18. S. Wongkaew, M. Caponigro, and A. Borzì, On the control through leadership of the hegselmannkrause opinion formation model, Mathematical Models and Methods in Applied Sciences, vol. 25, no. 03, pp. 565-585, 2015.

19. J. Lorenz, A stabilization theorem for dynamics of continuous opinions, Physica A: Statistical Mechanics and its Applications, vol. 355, no. 1, pp. 217-223, 2005.

20. J. M. Hendrickx, G. Shi, and K. H. Johansson, Finite-time consensus using stochastic matrices with positive diagonals, IEEE Transactions on Automatic Control, vol. 60, no. 4, pp. 1070-1073, 2015.

21. L. Radford, Three key concepts of the theory of objectification: Knowledge, knowing, and learning, Journal of Research in Mathematics Education, vol. 2, no. 1, pp. 7-44, 2013.

22. G. Williams and P. Liljedahl, Researching 'thinking classrooms', in Proceedings of the 38th Conference of the International Group for PME and the 36th Conference of the North American Chapter of the PME (Vol. 1), p. 249, Citeseer, 2014.

23. M. Huberman, The model of the independent artisan in teachers' professional relations, Teachers' work: Individuals, colleagues, and contexts, pp. 11-50, 1993.

24. A. H. Schoenfeld, Toward professional development for teachers grounded in a theory of decision making, ZDM, vol. 43, no. 4, pp. 457-469, 2011.

25. C. Andrà, N. Parolini, and M. Verani, BetOnMath. Azzardo e matematica a scuola. Springer-Verlag Italia, 2016.

26. D. B. McLeod, Research on affect in mathematics education: A reconceptualization, Handbook of research on mathematics teaching and learning, pp. 575-596, 1992.

27. A. H. Schoenfeld, Chapter 2: On modeling teachers' in-the-moment decision making, Journal for Research in Mathematics Education. Monograph, vol. 14, pp. 45-96, 2008.

28. A. H. Schoenfeld, Toward a theory of teaching-in-context, Issues in Education, vol. 4, no. 1, pp. 1-94, 1998. 